\title{
Editorial
}

\section{Fractional Integral Transform and Application}

\author{
Hassan Eltayeb, ${ }^{1}$ Adem Kilicman, ${ }^{2}$ and Mohamed Boussairi Jleli ${ }^{1}$ \\ ${ }^{1}$ Department of Mathematics, College of Science, King Saud University, P.O. Box 2455, Riyadh 11451, Saudi Arabia \\ ${ }^{2}$ Department of Mathematics and Institute for Mathematical Research, Universiti Putra Malaysia, 43400 Serdang, Selangor, Malaysia
}

Correspondence should be addressed to Hassan Eltayeb; hgadain@ksu.edu.sa

Received 8 December 2014; Accepted 8 December 2014

Copyright (C) 2015 Hassan Eltayeb et al. This is an open access article distributed under the Creative Commons Attribution License, which permits unrestricted use, distribution, and reproduction in any medium, provided the original work is properly cited.

It is well known that the methods connected to the employment of integral transforms are very useful in mathematical analysis. Those methods are successfully applied

(i) to solve differential and integral equations,

(ii) to study special functions,

(iii) to compute integrals.

In this special issue we publish many articles of the highest quality. The aim of these papers is to highlight the importance of fractional operators of integral transform and their applications and let the readers of this journal know about the possibilities of this new tool. In particular, various potential topics are discussed: (i) mathematical analysis of fractional integral transform theoretical methods, (ii) new methods for solving Cauchy problem for the space-time fractional partial differential equation, (iii) applications of integral transform fractional, including fractional methods, (iv) applying fractional integral transform to solution of systems of differential equations and applications in physics, mechanics, and fractional Schrödinger equation in quantum theory, (v) applications of integral transform for special functions, for example, prefunction, and (vi) applications of integral transform to fractional convolution products.

As editors of this special issue, the most difficult problem that we came across was to choose the best papers from the submitted high-quality works. All the papers published in this special issue are original and contain some attractive, resourceful, and recognizable ideas. Our motivation in choosing articles for publication in this special issue was whether the submitted papers activate and inspire further scientific activities in the scope of the special issue.

Hassan Eltayeb Adem Kilicman Mohamed Boussairi Jleli 


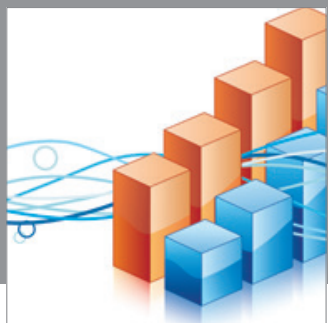

Advances in

Operations Research

mansans

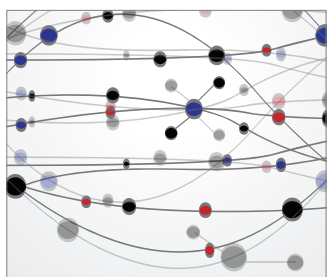

The Scientific World Journal
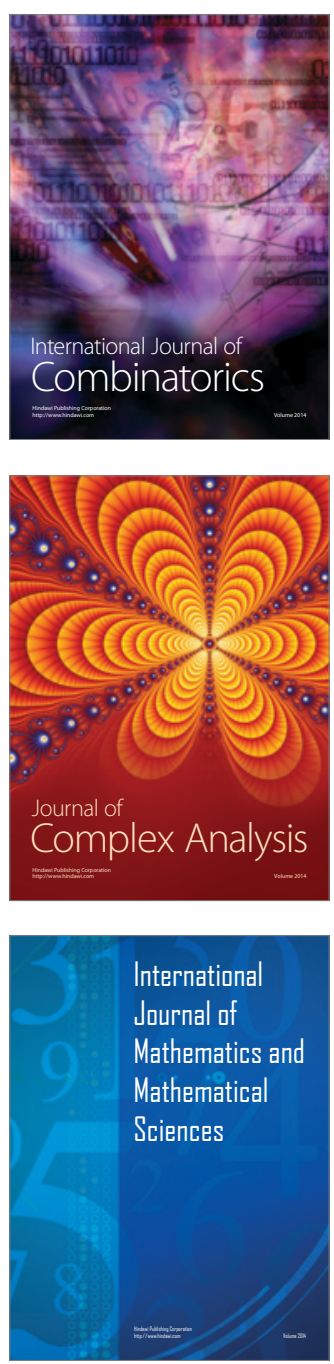
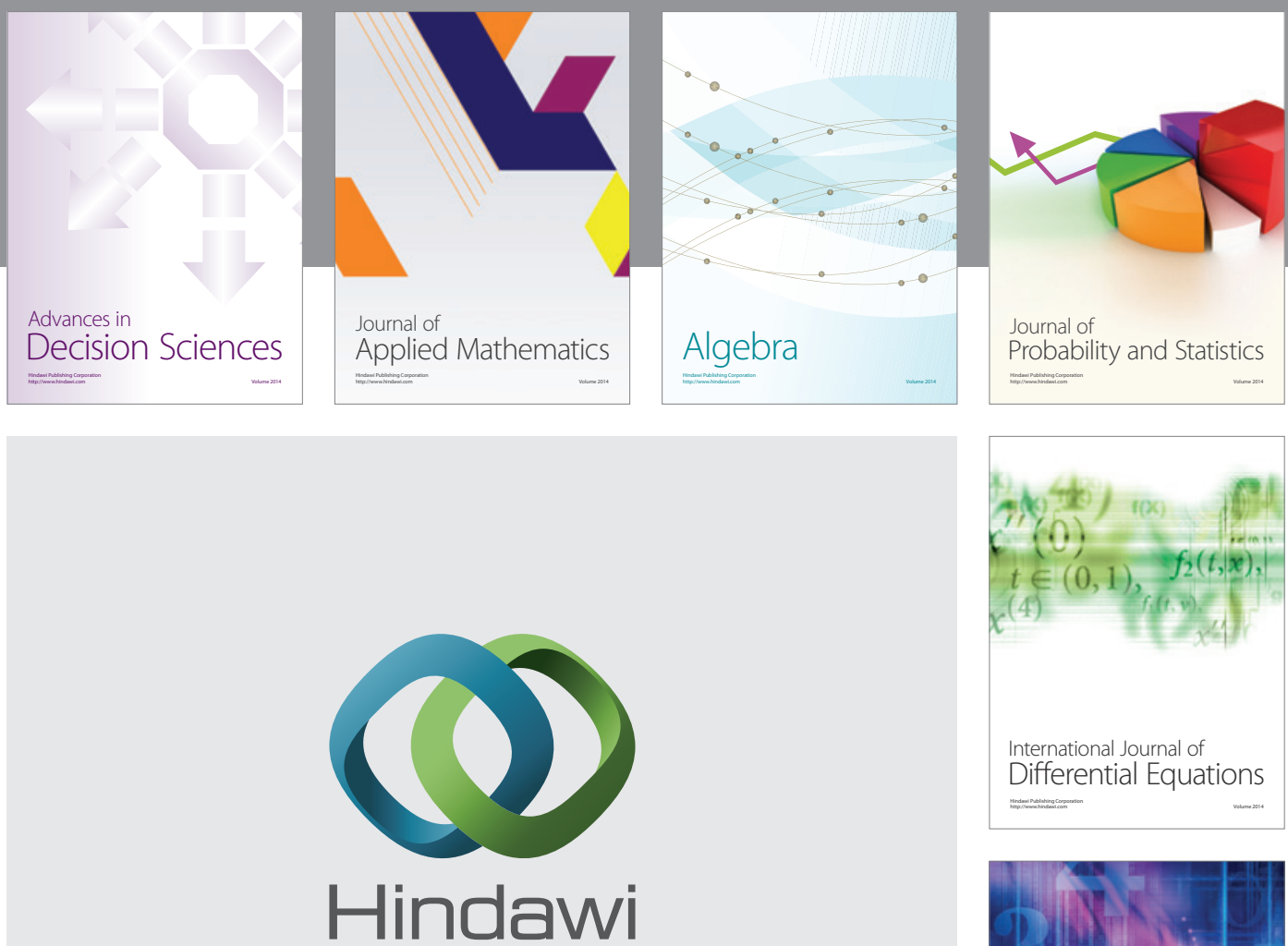

Submit your manuscripts at http://www.hindawi.com
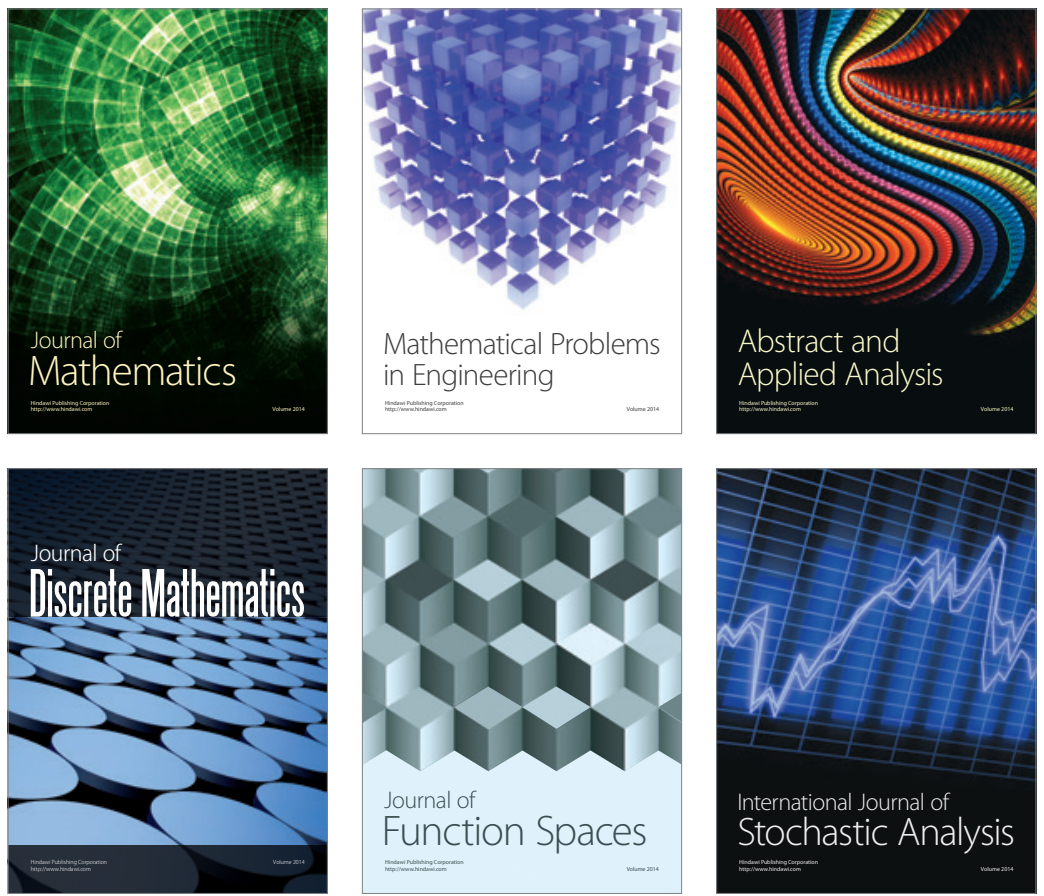

Journal of

Function Spaces

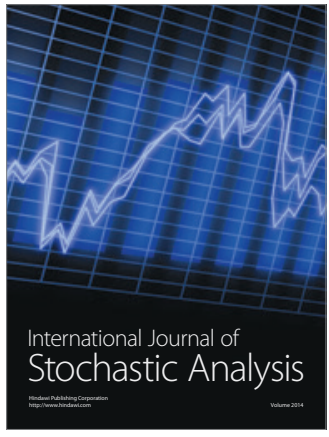

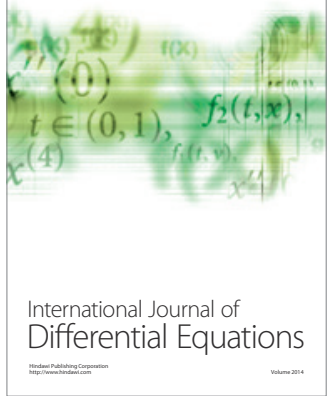
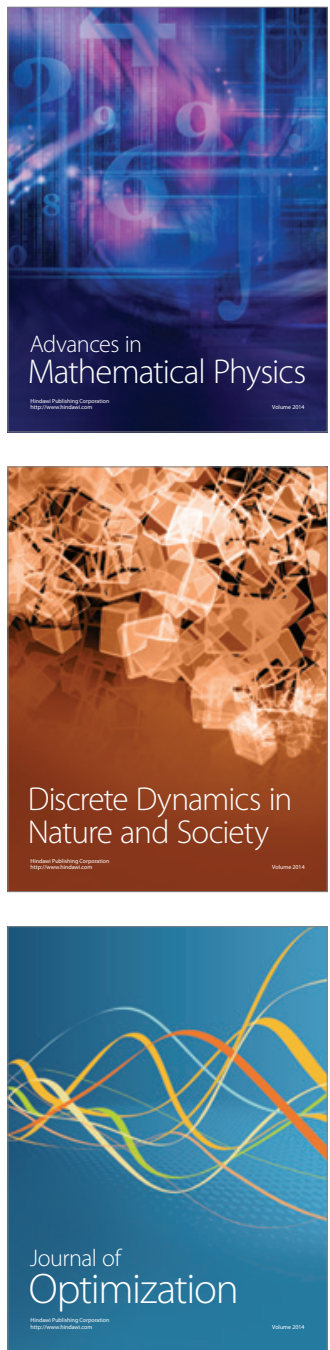